\title{
Neural representation of a spatial odor memory in the honeybee mushroom body
}

\author{
Martin P Nawrot ${ }^{1,2^{*}}$, Tiziano D'Albis ${ }^{1}$, Randolf Menzel ${ }^{3}$, Martin Strube-Bloss ${ }^{4}$ \\ From 24th Annual Computational Neuroscience Meeting: CNS*2015 \\ Prague, Czech Republic. 18-23 July 2015
}

Insects make use of lateralized olfactory information from their left and right antennae. Honeybees can learn to distinguish side-specific odor cues in classical conditioning experiments, i.e. they associate a specific stimulus combination of odor identity and spatial location (left or right) with the reward [1]. This requires interhemispheric transfer of lateralized information and a side-specific odor memory. Mushroom body (MB) output neurons, the so-called extrinsic neurons (ENs), make inter-hemispheric connections between the two MBs and are thus candidates for the inter-hemispheric transfer of lateralized stimulus information. We could show previously that ENs in the honeybee undergo plastic changes in classical conditioning [2].

Here, we investigate neuronal plasticity in ENs of the honeybee in a sides-specific learning paradigm. We performed multiple single-unit recordings from ENs of one MB. Prior to conditioning (PRE) each bee was repeatedly presented with two different odors on the two antennae separately. During acquisition one of these odors was repeatedly presented to the antenna contralateral to the recording side $(\mathrm{CS}+)$ and paired with a sugar reward, while the other odor was presented without reward (differential conditioning). Three hours after training (POST) we repeated the initial protocol presenting each of the two odors repeatedly to each of the antennae. In the behavioral test the bees distinguished the CS+ stimulus configuration of odor and side from the other three stimulus combinations.

At the neuronal level we found clear and distinct odor representations in the EN population before training (PRE) only when stimulated on the antenna ipsilateral to the recording side. However, no odor responses were measured in any of the ENs when stimulated at the

\footnotetext{
* Correspondence: martin.nawrot@fu-berlin.de

${ }^{1}$ Bernstein Center for Computational Neuroscience Berlin, Berlin, Germany Full list of author information is available at the end of the article
}

contralateral antenna. This picture changed drastically after training (POST). Now, the rewarded stimulus combination $\left(\mathrm{CS}_{+}\right)$resulted in a strong population response pattern. The population code for the CS+ configuration was clearly distinct from all three other stimulus configurations. Quantification of the temporal response latencies showed that the ENs encode an odor approximately within $75 \mathrm{~ms}$ for ipsilateral stimulation. Odor representation was delayed by about $60 \mathrm{~ms}$ for a contralateral stimulation. We discuss two alternative explanations for this temporal delay.

We hypothesized previously that ENs at the MB output encode the experience-dependent value of a particular stimulus [2-4]. Our results here provide additional evidence for this hypothesis. A representation of the rewarded stimulus combination (CS+) of a particular odor and its spatial location (left or right) develops only due to reward conditioning. Before learning, only ipsilateral odor information was represented.

\section{Acknowledgements \\ Funding was received from the Federal Ministry of Education and Research (BMBF) within the Bernstein Focus Neuronal Basis of Learning (BFNL) through grant 01GQ0941 to M.N. and R.M.}

\footnotetext{
Authors' details

${ }^{1}$ Bernstein Center for Computational Neuroscience Berlin, Berlin, Germany. ${ }^{2}$ Computational Systems Neuroscience, Department of Biology, University of Cologne, Cologne, Germany. ${ }^{3}$ Institute of Biology - Neurobiology, Freie Universität Berlin, Berlin, Germany. ${ }^{4}$ Department of Behavioral Physiology \& Sociobiology, Biocenter, University of Würzburg, Würzburg, Germany.

Published: 18 December 2015
References
1. Sandoz JC, Menzel R: Side-Specificity of Olfactory Learning in the Honeybee: Generalization between Odors and Sides. Learning \& Memory 2001, 8:286-294. encode odor-reward associations. J Neurosci 2011, 31:3129-3140. recoding device. J Physiol Paris 2014, 108:84-95.

2. Strube-Bloss MF, Nawrot MP, Menzel R: Mushroom body output neurons

3. Menzel R: The insect mushroom body, an experience-dependent
} 
4. Kloppenburg P, Nawrot MP: Neural Coding: Sparse but On Time. Current Biology 2014, 24:R957-R959.

doi:10.1186/1471-2202-16-S1-P240

Cite this article as: Nawrot et al:: Neural representation of a spatial odor memory in the honeybee mushroom body. BMC Neuroscience 2015 16(Suppl 1):P240.
Submit your next manuscript to BioMed Central and take full advantage of:

- Convenient online submission

- Thorough peer review

- No space constraints or color figure charges

- Immediate publication on acceptance

- Inclusion in PubMed, CAS, Scopus and Google Scholar

- Research which is freely available for redistribution

Submit your manuscript at www.biomedcentral.com/submit
C Biomed Central 